\title{
Autoeficácia materna no cuidado do recém-nascido prematuro: utilização da técnica brainstorming
}

\author{
Maternal self-efficiency in premature newborn care: use of the brainstorming technique \\ Autoeficiencia materna en el cuidado de recién nacidos prematuros: uso de la técnica de \\ brainstorming
}

Nalma Alexandra Rocha de Carvalho Poty ${ }^{1 *}$, Silvana Santiago da Rocha ${ }^{2}$, Raiana Soares de Sousa e Silva ${ }^{2}$, Damila Rufino de Holanda e Silva ${ }^{2}$, Roama Paulo Ulisses Vaz da Costa ${ }^{1}$, Joaquim Guerra de Oliveira $\mathrm{Neto}^{3}$, Bárbara de Jesus Cunha da Silva ${ }^{2}$, Kassya Fernanda Freire ${ }^{4}$, Jéssica de Moura Caminha², Shelma Feitosa dos Santos ${ }^{5}$.

\section{RESUMO}

Objetivo: Levantar as principais dificuldades acerca dos cuidados aos recém-nascidos prematuros após a alta hospitalar, por meio de um diagnóstico situacional, utilizando a técnica brainstorming para o levantamento das informações. Métodos: Trata-se de uma pesquisa do tipo descritiva com característica qualitativa, utilizando como procedimento para coleta de dados a técnica brainstorming para levantar as principais dificuldades acerca dos cuidados aos recém-nascidos prematuros após a alta hospitalar. O período que ocorreu a pesquisa foi maio de 2019. Resultados: Ao questionar as mães sobre os cuidados domiciliares após a alta do seu filho prematuro, surgiu inicialmente sentimentos de alegria, em alguns momentos sentimentos de medo em relação ao cuidado e fragilidade do filho, dessa forma a alegria se misturou ao medo de cuidar do filho prematuro em casa. $O$ resultado deste brainstorming gerou respostas que envolveram diversos eixos do cuidado, estas relacionadas a higiene, alimentação, crescimento e desenvolvimento, cuidados de rotina, cuidados especiais, prevenção de doenças e vacinas. Conclusão: Foi evidenciado ainda que, para as mães, o momento da alta de seus filhos está relacionado a diversos sentimentos, entre eles está o medo, a angústia, sensação de fragilidade, insegurança, além dos desafios que virão.

Palavras-chave: Alta do paciente, Unidades de terapia intensiva neonatal, Prematuro.

\begin{abstract}
Objective: To raise the main difficulties regarding the care of premature newborns after hospital discharge, through a situational diagnosis, using the brainstorming technique to gather information. Methods: This is a descriptive research with a qualitative characteristic, using the brainstorming technique for data collection to raise the main difficulties regarding the care of premature newborns after hospital discharge. The research period was May 2019. Results: When questioning mothers about home care after the discharge of their premature child, feelings of joy initially arose, in some moments feelings of fear in relation to the care and fragility of the child, in this way the joy was mixed with the fear of taking care of the premature child at home. The result of this brainstorming generated responses that involved several axes of care, these related to hygiene, food, growth and development, routine care, special care, disease prevention and vaccines. Conclusion: It was also evidenced that the period of discharge of the premature baby for mothers is permeated with multiple feelings such as anguish, fragilities, insecurity, fears and challenges. In this sense, health professionals have a fundamental role in building the link between mothers and babies, seeking to build autonomy for maternal care at home after hospital discharge.
\end{abstract}

Keywords: Patient discharge, Neonatal intensive care units, Premature.

\footnotetext{
${ }^{1}$ Universidade Federal do Maranhão (UFMA), São Luís - MA. *E-mail: enf.nalma.carvalho@hotmail.com

2 Universidade Federal do Piauí (UFPI), Teresina - PI.

${ }^{3}$ Universidade Federal do Tocantins (UFT), Araguaína - TO.

${ }^{4}$ Faculdade Santa Terezinha (CEST), Teresina - PI.

${ }^{5}$ Centro Universitário Uninovafapi (UNINOVAFAPI), Teresina - PI.
} 


\section{RESUMEN}

Objetivo: Plantear las principales dificultades en la atención del recién nacido prematuro tras el alta hospitalaria, a través de un diagnóstico situacional, utilizando la técnica del brainstorming para recabar información. Métodos: Se trata de una investigación descriptiva con carácter cualitativo, utilizando la técnica de lluvia de ideas para la recolección de datos para plantear las principales dificultades en la atención al recién nacido prematuro después del alta hospitalaria. El período de investigación fue mayo de 2019. Resultados: Al cuestionar a las madres sobre el cuidado domiciliario luego del alta de su hijo prematuro, inicialmente surgieron sentimientos de alegría, en algunos momentos sentimientos de miedo en relación al cuidado y fragilidad del niño, de esta manera la alegría se mezcló con el miedo de cuidar. del niño prematuro en casa. El resultado de esta lluvia de ideas generó respuestas que involucraron varios ejes de atención, estos relacionados con la higiene, alimentación, crecimiento y desarrollo, cuidados de rutina, cuidados especiales, prevención de enfermedades y vacunas. Conclusión: También se evidenció que el período de alta del prematuro para las madres está impregnado de múltiples sentimientos como angustias, fragilidades, inseguridad, miedos y desafíos. En este sentido, los profesionales de la salud tienen un papel fundamental en la construcción del vínculo entre madres y bebés, buscando construir la autonomía para el cuidado materno en el hogar después del alta hospitalaria.

Palabras clave: Alta del paciente, Unidades de cuidados intensivos neonatales, Prematuro.

\section{INTRODUÇÃO}

No ambiente de cuidados neonatais, é importante considerar os impactos que a hospitalização traz à família do Recém-nascido (RN). O nascimento do RN prematuro e de baixo peso implica em mudanças nos planos familiares, por causar frustrações em relação ao que foi idealizado pelos pais: uma criança bonita, saudável, com peso adequado e que fosse direto para casa. Para as puérperas, receber alta sem o seu filho gera um sentimento de frustração. Dessa forma, o parto prematuro e a necessidade de internação do RN representam um momento marcante na vida de uma mulher (SALES IMM, et al., 2018).

Ressalta-se que a prematuridade é a primeira causa de internações em Unidade de Terapia Intensiva Neonatais (UTIN), devido a isso o momento da alta hospitalar é um período no qual as orientações direcionadas às necessidades desse $\mathrm{RN}$ em domicílio são imprescindíveis. Esse momento é espero e ao mesmo tempo estressante para os pais, rodeado de esperanças e incertezas, pois é nesse momento que os pais e os familiares tomam verdadeiramente os cuidados com o seu filho (ALCÂNTARA KL, et al., 2017).

A alta hospitalar é motivo de grande expectativa por parte dos pais, pois após um longo período de internação, com melhoras significantes do estado de saúde do filho, a família aguarda esse momento, permeado de sentimentos como tranquilidade, alegria e ansiedade em busca do dia esperado (SOUZA ML, et al., 2018).

Esse processo de alta do prematuro da unidade neonatal é complexo, pois os pais vivenciam sentimentos conflitantes. Por isso, a enfermagem deve desenvolver estratégias que envolvam os pais desde o início da internação no cuidado de seus filhos, para adquirir confiança nesses cuidados (OSORIO G, et al., 2017). E assim se apropriará de meios adequados para cuidar do neonato com destreza e segurança (ROCHA GMN, et al., 2019).

Ressalta-se a relevância da Política Nacional de Atenção Integral à Saúde da Criança na educação em saúde dada as mães de prematuros. Uma vez reconhecida a prematuridade como prevalente no contexto da saúde pública e o reconhecimento de suas consequências para a saúde, essa política traz em seus eixos estruturantes orientações para a vigilância do crescimento e desenvolvimento infantil, amamentação e atenção integral a crianças em situações de agravos e vulnerabilidades, desafios no seguimento qualificado de crianças nascidas prematuras (SILVA CSSL, et al., 2018).

É necessário conhecer também as vivências dos pais para entender melhor suas necessidades e como podem ser mais bem acompanhados durante o processo de alta. É importante gerar estratégias que potencializem o processo de preparação para alta dos pais de bebês prematuros, para que estes diminuam - estresse e aumentem a sensação de segurança e confiança na alta do filho da unidade neonatal. Impactando positivamente na qualidade do cuidado ao prematuro em casa (OSORIO G, 2017). 
Evidencia-se que conhecimento e a compreensão da vivência de mães de prematuros nos primeiros meses no domicílio após a alta hospitalar podem auxiliar os profissionais de saúde a entender de forma holística todos os sentimentos que envolvem esse processo e assim desenvolver estratégias educativas e intervenções de aconselhamento de acordo com as necessidades maternas. A prática e os programas de alta precoce podem facilitar o processo de alta. A promoção do cuidado domiciliar do prematuro requer a participação de toda a equipe neonatal, especificamente dos profissionais de enfermagem, que prestam assistência contínua (MOLINA JG, et al., 2019; ROCHA GMN, et al., 2019).

Com base no acima exposto, esse trabalho objetiva levantar as principais dificuldades acerca dos cuidados aos recém-nascidos prematuros após a alta hospitalar, por meio de um diagnóstico situacional, utilizando a técnica brainstorming para o levantamento das informações.

\section{MÉTODOS}

Trata-se de uma pesquisa do tipo descritiva com característica qualitativa, utilizando como procedimento para coleta de dados a técnica brainstorming para levantar as principais dificuldades acerca dos cuidados aos RNs prematuros após a alta hospitalar. O período que ocorreu a pesquisa foi maio de 2019.

A técnica brainstorming. foi criada por Alex Osborn, em 1953, cuja tradução significa "tempestade de ideias". Os autores enfatizaram que este método é muito utilizado em diversas áreas de pesquisa devido à sua simplicidade (ALVES HÁ, et al., 2007). A técnica de brainstorming tem como objetivo coletar ideias de forma coletiva, sem críticas ou julgamentos. Com isso, a escolha pela técnica também se justifica pelo fato de os participantes deste estudo possuírem baixo nível de escolaridade (NÓBREGA MM, et al., 1997).

De acordo com Masseto MT (2003) tempestade de ideias ou brainstorming trata-se de uma técnica que permite o desenvolvimento da criatividade e a produção de uma grande quantidade de ideias em um curto espaço de tempo.

A coleta de dados se deu por meio da aplicação de questionários e observações in loco. O diagnóstico situacional pode ser entendido como o resultado de um processo de coleta, tratamento e análise dos dados colhidos no local onde se deseja realizá-lo. Este pode ser considerado como uma das mais importantes ferramentas de gestão, constituído por pesquisa das condições de saúde e risco de uma determinada população, para posterior planejamento e programação das ações (SILVA CSSL, 2016), através deste estabelecem-se as prioridades de acordo com a realidade da instituição, da população e do território.

Metodologicamente, o processo de brainstorming segue as seguintes fases: 1 Fase: Criativa - os participantes da sessão apresentam o maior número de ideias e sugestões sem se preocuparem em analisálas ou criticá-las; $2^{\circ}$ Fase: Crítica - os participantes da sessão, individualmente, justificam e defendem suas ideias; é a fase de filtração de ideias para a permanência das que foram melhor fundamentadas e de aceitação do grupo (NÓBREGA MM, et al., 1997).

Dessa forma a pesquisa (brainstorming) aconteceu na Unidade de Cuidados Intermediários Canguru (UCINCa) de uma Maternidade de referência do estado do Piauí, conhecida como "Enfermarias Canguru". A coordenadora foi a pesquisadora participante (mestranda), o secretário: outro mestrando do grupo de estudo do pesquisador responsável pela pesquisa e os membros foram o público alvo: doze mães de prematuros internados em duas enfermarias do referido setor.

Inicialmente a coordenadora e o secretário se apresentaram, organizaram o ambiente (em forma de círculo), convidaram as mães a participar, explicaram o objetivo da atividade para então serem levantadas as informações, baseado no tema (cuidados aos prematuros após alta hospitalar: o que vocês ainda precisam saber?), assim houve a discussão e conclusões oportunas.

Os critérios de inclusão foram: mães com filhos internados na UCINCa há mais de 48h (devido a melhor entendimento do processo de cuidado), e os critérios de exclusão desta pesquisa foram mães com déficit cognitivo motor que impossibilitasse a compreensão das informações repassadas.

Esse trabalho foi submetido ao Comitê de Ética em Pesquisa (CEP) da Universidade Federal do Piauí (UFPI) que obteve aprovação por meio do parecer ํo. 2.883.281 e Certificado de Apresentação para Apreciação Ética, com CAAE no. 96521118.3.0000.5214. 
Destaca-se que foram obedecidos pelos pesquisadores os princípios da ética, sigilo e confidencialidade. Aos participantes (público alvo) foi apresentado o Termo de Consentimento Livre e Esclarecido, garantindo a confidencialidade, a privacidade, e, ainda, a não utilização das informações em prejuízo dos participantes da pesquisa, conforme Resolução no. 466/12 do Conselho Nacional de Saúde (CNS), do Ministério da Saúde, que trata dos procedimentos éticos das pesquisas envolvendo seres humanos.

\section{RESULTADOS}

Ao questionar as mães sobre os cuidados domiciliares após a alta do seu filho prematuro, surgiu inicialmente sentimentos de alegria, em alguns momentos sentimentos de medo em relação ao cuidado e fragilidade do filho, dessa forma a alegria se misturou ao medo de cuidar do filho prematuro em casa.

O resultado deste brainstorming gerou respostas que envolveram diversos eixos do cuidado, estas relacionadas a higiene, alimentação, crescimento e desenvolvimento, cuidados de rotina, cuidados especiais, prevenção de doenças e vacinas (Figura 1).

Figura 1 - Contribuicões levantadas pelas mães de prematuros internados acerca dos cuidados domiciliares. banho (frequência, produtos) frequência de amamentação vômitos alimentação complementar alimentação da mãe engasgamentos

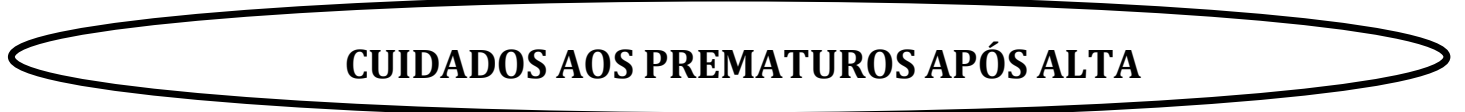

identificação de problemas respiratórios cuidados com visitas higiene das mãos

vacinas (época correta) marcos do desenvolvimento (como estimular)

sono (tempo necessário, como acordar, local adequado) temperatura consultas

Fonte: Poty NARC, et al., 2021.

Pela análise das respostas obtidas, observa-se que, as mães ainda possuíam muitas dúvidas, principalmente a respeito da higiene, alimentação, cuidados diários e intercorrências, mesmo estando 24 horas em contato com seus filhos e realizando o processo do cuidado durante o período de internação, ainda assim a situação da alta ainda causava medo e preocupação. Uma primeira leitura dos dados acima traz a existência de ideias que vão desde cuidados simples (temperatura do ambiente) até possíveis intercorrências (engasgo, problemas respiratórios), algumas dúvidas eram parciais ou apenas com necessidade de confirmação, outras se mostravam com total desconhecimento (calendário vacinal para RNs prematuros, manobras de engasgamento).

Da análise dos dados emergiram os seguintes temas que posteriormente foram utilizados para elaboração de uma cartilha educativa: 1. Higiene - Banho Coto umbilical Lavagem de roupas Limpeza da casa e roupas de cama Limpeza de utensílios (copinhos, mamadeira, brinquedos, etc.); 2. Alimentação - Aleitamento Materno Alimentação Complementar; 3. Crescimento e desenvolvimento - Como estimular seu filho; 4. Cuidados diários - Choro Sono Sol Frio e calor Visitas Medicações e vitaminas; 5. Cuidados especiais/intercorrências - Nariz entupido Engasgo Vômitos e regurgitação; e 6. Consultas e vacinas.

Com a realização da atividade de brainstorming conseguiu-se, numa perspectiva aberta e de diálogo, conhecer um pouco melhor a realidade das mães de RNs prematuros, tanto ao nível dos conhecimentos como das habilidades face aos cuidados domiciliares aos seus filhos. Os dados recolhidos permitiram-nos verificar que, as mães, embora conscientes do que vão enfrentar após a alta, ainda desconhecem muitos cuidados a serem desempenhados por elas. 


\section{DISCUSSÃO}

O cuidado domiciliar ao prematuro egresso de Unidade de Terapia Intensiva Neonatal (UTIN) é permeado por sentimentos contraditórios, pois as cuidadoras afirmam desejar estar em casa com seus filhos, mas relatam sentir insegurança, medo de assumir o cuidado com o prematuro que vivenciou um processo de internação prolongado e demandou cuidados específicos. Aliado a isto, há uma reorganização do cotidiano das cuidadoras para atendimento das demandas do prematuro e elas reconhecem que este exige uma atenção diferenciada para prevenir adoecimentos. Mesmo expressando dificuldades e mudanças, no cotidiano da vida, as mães afirmam assumir sozinhas as responsabilidades com o cuidado de seus filhos (BRAGA PP; SENA RR, 2017).

As famílias vivenciam o cuidado de suas crianças no contexto doméstico como uma adaptação processo, permeado por sentimentos de alegria, alívio, insegurança e medo. A chegada da criança em casa gera mudanças na organização familiar e torna-se o foco da família, que se esforça para prestar os cuidados necessários. A proximidade com a criança após a alta é um ponto positivo relatado por os pais (FELIZARDO MJA, et al., 2020).

A falta de experiência para o cuidado remete à insuficiência do estoque de conhecimento sobre as intercorrências comuns apresentadas pelo RN. Essa situação pode ter como repercussão o aumento do estresse materno, uma vez que as memórias do estresse vivido na hospitalização ainda são recentes, o que poderá comprometer 0 desenvolvimento da competência materna saudável. Embora a prematuridade represente para as mães um momento de estresse, que lhes incita medos e inseguranças, com a adaptação materna ao cuidado do filho em casa, com apoio da família e com o desenvolvimento de seu papel materno, passa a vislumbrar um futuro para o filho (MACHINESKI GG, et al., 2018).

Um estudo demostrou que os pais de bebês prematuros extremos tiveram um impacto negativo maior na Qualidade de Vida Relacionada à Saúde (QVRS) durante a internação na UTIN e mais melhora após a alta do que os pais de outros bebês hospitalizados na UTIN. Cuidados domiciliares complexos foram associados com menor QVRS dos pais após a alta. O benefício potencial da alta domiciliar deve ser equilibrado em relação ao impacto negativo potencial de cuidados domiciliares complexos (MCANDREW S, et al, 2019).

Semelhantes aos resultados encontrados, um estudo acerca dos cuidados domiciliares com o RN prematuro realizou um storyboard, no qual aborda a relevância da higienização das mãos, da não aproximação do bebe em caso de doença infectocontagiosa, de diminuir as visitações e da importância da vacinação. Ele aborda ainda a necessidade de conservar o ambiente limpo, arejado, e sem umidade, aborda ainda a necessidade de higienizar as cortinas com constância e evitar deixar bichos de pelúcia juntos ao RN. Recomenda evitar ruídos e luz, assim como não fumar no ambiente que o RN estiver e realizar medidas que evitem o risco de morte súbita (PINTO TRC, et al., 2018).

Sobre a amamentação, foi evidenciado e um estudo que acontece a redução significativa na prevalência aleitamento materno exclusivo ao longo do primeiro mês no domicílio e que o acompanhamento nessa fase é fundamental para se evitar o desmame precoce (LIMA AAE, et al., 2019).

Ainda acerca da amamentação, é importante acordar a criança para amamentar, tendo em vista que o prematuro é mais sonolento, para acorda-lo pode-se retirar as roupas lentamente, mexer no rosto do bebe, conversar. Ressalta-se a importância da pega correta e de aprender as posições de amamentação, para que a mãe tenha conhecimento e possa utiliza-las. Sobre intercorrências, deve-se atentar acerca dos casos de engasgo durante a alimentação, observar cor da pele, presença de cianose, e aprender a manobra a ser utilizada para desobstrução das vias aéreas superiores. Quando ocorrer, deve-se posicionar a cabeça do RN lateralmente para drenar o alimento para a boca, impedindo que o alimento regresse para o pulmão (PINTO TRC, et al., 2018).

Um estudo realizado em Sobral- CE, dentro do contexto do Projeto Coala, que tem como proposta garantir a alta precoce das crianças prematuras e com Crescimento Intrauterino Restrito (CIUR) comprovou que as principais dificuldades dos cuidadores estavam geralmente relacionadas à insegurança no cuidar e o medo de que algo pudesse acontecer com o bebê. Dos 13 participantes, 10 relataram algum tipo de dificuldade 
como: o medo de manusear durante o banho e a troca, de não conseguir compreender o motivo do choro da criança, de administrar medicações via oral e nasal, da adaptação aos horários da alimentação, da forma como alimentar e da dificuldade por parte dos cuidadores, em dormirem, por ficarem normalmente em alerta e observando a criança dormir (ARCANJO CCT, et al., 2018).

Quanto às situações de emergência, o mesmo estudo demostrou que os cuidadores após a alta vivenciaram alguma circunstância adversa com a sua criança, e a mais mencionada foi o engasgamento. $A$ prevalência desse acidente se dá pelo refluxo gastresofágico muito comum em recém-nascido prematuro, a dificuldade de deglutição e a facilidade de broncoaspiração. É necessário, nesses casos, ações rápidas que previnam asfixia e morte súbita (ARCANJO CCT, et al., 2018).

Em relação ao sono do $\mathrm{RN}$, uma pesquisa ressaltou a importância de um ambiente tranquilo, com pouca luz para que o bebê consiga dormi tranquilamente. Além disso, a posição adequada para dormir deve ser em decúbito dorsal para que não ocorra a síndrome de morte súbita do lactente, e orienta ainda a não o colocar para dormir na cama junto com os pais, para não causar acidentes. E importante reconhecer a comunicação não verbal dos RNs pelos pais e cuidadores, sabendo interpretar os diferentes tipos de choro. Além disso, deve-se reservar um tempo para atividades de lazer: para passear, tomar banho de sol e brincar com o filho, tais atitudes contribuem para o desenvolvimento neuropsicomotor (PINTO TRC, et al., 2018).

Sobre cuidados com a higiene, especificadamente o banho gera nas mães muita expectativa gerando assim inseguranças no momento desse simples ato, por medo de cair, por conta da presença do coto umbilical e à fragilidade da criança acaba que contribuem para essa insegurança materna. Assim, as orientações desses cuidados destinados às mães devem ser repetidas várias vezes para que sejam absorvidos e colocados em prática, até que a mãe se sinta segura para a sua realização. O profissional de saúde deve orientar a mãe e acompanhar a evolução da mesma, levando em consideração a autonomia e confiança da mesma, inicialmente orientando, posteriormente, auxiliando a mãe no cuidado, e finalmente, supervisionando a realização do procedimento, continuamente de forma disponível e acolhedora (VERONEZ M, et al., 2017).

A experiência dos pais de receberem alta para casa com um bebê prematuro pode ser emocionalmente desafiadora e requer uma série de mecanismos de apoio para ajudá-los a lidar com esse período de transição. Os profissionais de saúde podem direcionar os pais aos serviços de aconselhamento, recursos e grupos de apoio de pares apropriados. Educação personalizada é necessária para profissionais de saúde baseados na comunidade, como visitantes de saúde, para equipá-los para apoiar os pais de forma prática e emocional durante a transição para casa e depois. Além disso, os profissionais de saúde podem aprender muito com os pais sobre o que é necessário para apoiá-los (PETTY J, et al., 2018).

A adaptação ao papel da maternidade pode ser promovida por meio do apoio às mães de bebês prematuros, capacitando-as e aumentando sua autoeficácia, para eliminar fatores e emoções negativas (HEYDARPOUR S; KESHAVARZ Z; BAKHTIARI M, 2017).

A vivência diária com prematuro é importante para a confiança e habilidades maternas sejam efetivadas. Os cuidados realizados ainda durante a internação neonatal são fundamentais para o preparo das mães nesse desafio do cuidado no domicílio, além de proporcionar ao profissional, a chance de avaliar se o cuidado prestado e as orientações educativa dadas durante toda internação estão sendo administrados de forma apropriada (VERONEZ M, et al., 2017).

É preciso que haja um planejamento de alta, que envolva mãe-família e equipe multidisciplinar, e uma rede de atenção à saúde eficiente, com ações articuladas entre a unidade hospitalar de referência e o serviço de atenção básica que garantam o cuidado integral por meio da oferta de serviços contínuos nos diferentes níveis de complexidade (LIMA AAE, et al., 2019).

Vivenciar um parto prematuro traz novas requisições, muitas vezes desconhecidas para o enfrentamento da família à nova situação. Recursos afetivos precisam ser fortalecidos, competências precisam ser aprendidas, habilidades necessitam ser modeladas. Os pais/família precisam do suporte das equipes em todas as esferas de atendimento, tanto hospitalar, quanto ambulatorial e até mesmo domiciliar. As orientações transmitidas pelos profissionais devem ser de fácil compreensão, esclarecedoras e de acordo com a realidade que as mães vivenciaram com o filho durante a internação hospitalar (BUSATTO E, et al., 2021). 


\section{CONCLUSÃO}

Os principais assuntos que as mães requerem informação são acerca da higiene, alimentação, crescimento e desenvolvimento, cuidados de rotina, cuidados especiais, prevenção de doenças e vacinas. Foi evidenciado ainda que, para as mães, o momento da alta de seus filhos está relacionado a diversos sentimentos, entre eles está o medo, a angústia, sensação de fragilidade, insegurança, além dos desafios que virão. Assim, os profissionais que atuam diretamente com esse público são fundamentais para a construção desse elo mãe- bebê ainda no período de internação, onde será construída a autoeficácia materna para o cuidado no domicílio pós alta hospitalar. Ressalta-se ainda a relevância das orientações e acompanhamento continuado dessas mães desde a internação, sendo preparadas para a alta, sendo trabalhada a sua individualidade e suas condições de moradia, rede de apoio, para assim se apropriarem dos cuidados e procedimentos a serem realizados em seus filhos, garantindo segurança e confiança no cuidado domiciliar.

\section{REFERÊNCIAS}

1. ALCÂNTARA KL, et al. Family guidelines needed for a safe hospital of the premature newborn: integrative review. Rev Enferm UFPE[Internet], 2017; 11(2): 645-655.

2. ALVES HÁ, et al. Aplicação da técnica criativa Brainstorming Clássico na geração de alternativas na criação de games. In: SIMPÓSIO BRASILEIRO DE JOGOS PARA COMPUTADOR E ENTRETENIMENTO DIGITAL, 6., 2007, Porto Alegre. Anais [...] 2007. Disponível em: http://www.sbgames.org/papers/sbgames07/artanddesign/full/ad2.pdf. Acesso em: 07 jul. 2020.

3. ARCANJO CCT, et al. Vivências de cuidadores de crianças prematuras após alta hospitalar: experiência do projeto coala. Essentia (Sobral), 2018;19(1):76-85.

4. BRAGA PP, SENA RR. Devir cuidadora de prematuro e os dispositivos constituintes da continuidade da atenção pósalta. Texto contexto - enferm. [online]. 2017; 26(3): e3070016.

5. BUSATTO E. Cuidados com o recém-nascido após alta hospitalar: orientações aos pais. Research, Society and Development, 2021; 10(2): e30610212541.

6. FELIZARDO MJA, et al. Families' experiences in home care for premature children: a qualitative systematic review. Revista de Enfermagem do Centro-Oeste Mineiro, 2020; 10: e3906.

7. HEYDARPOUR S, et al. Factors affecting adaptation to the role of motherhood in mothers of preterm infants admitted to the neonatal intensive care unit: A qualitative study. Journal of advanced nursing, 2017; 73(1): 138-148.

8. LIMA AAE, et al. Aleitamento materno exclusivo de prematuros e motivos para sua interrupção no primeiro mês pósalta hospitalar. Rev. Gaúcha Enferm., 2019; 40(e20180406): 1-8.

9. MACHINESKI GG, et al. Percepção das mães quanto à competência materna nos cuidados domiciliares do recémnascido prematuro. Revista Saúde (Santa Maria), 2018; 44(3): 1-13.

10. MASSETO MT. Competências Pedagógicas do Professor Universitário. São Paulo: Summus, 2003.

11. MCANDREW S, et al. Prospective Study of Parent Health-Related Quality of Life before and after Discharge from the Neonatal Intensive Care Unit. The Journal of Pediatrics, 2019; 213(3): 38-45.

12. MOLINA JG, et al. Experiences of Mothers of Extremely Preterm Infants after Hospital Discharge. Journal of Pediatric Nursing, 2019; 45(1): 2-8

13. NÓBREGA MM, et al. Uso da técnica de brainstorming para tomada de decisões na equipe de enfermagem de saúde pública. R. Bras. Enferm., 1997; 50(2): 247-256.

14. OSORIO G, et al. Preparando-se para os cuidados pós-alta de bebês prematuros: Experiências de pais. Investir. educ. enferm [online], 2017; 35(1): 100-106.

15. OSORIO SP, et al. Preparando-se para o cuidado pós-alta ao prematuro: Experiências dos pais. Investir. Educ. Enferm., 2017; 35(1): 100-106.

16. PETTY J, et al. Parents' views on preparation to care for extremely premature infants at home. Nursing Cildren \& Young People, 2018; 30(4): 22-27

17. PINTO TRC, et al. Animação educativa sobre cuidados domiciliares com o prematuro. Rev. Bras. Enferm., 2018; 71(4): 1604-1610.

18. ROCHA GMN. Dúvidas maternas na alta hospitalar do recém-nascido. Revista Univap, 2019; 25(49): 93-103.

19. SALES IMM, et al. Sentimentos de mães na unidade canguru e as estratégias de suporte dos profissionais de enfermagem. Rev Cuid., 2018; 9(3): 2413-2422.

20. SILVA CSSL et al. O Diagnóstico Situacional como ferramenta para o planejamento de ações na Atenção Primária a Saúde. Revista Pró-UniverSUS, Vassouras. 2016; 7(2): 30-33.

21. SOUZA ML, et al. Repercussões no cuidado domiciliar para o pai participante do protocolo de assistência ao filho prematuro. REAS, Revista Eletrônica Acervo Saúde, 2018; 10(4): 1727-1734.

22. SUSAN L, et al. Information and Support Needs of Parents With Premature Infants: An Integrative Review. Journal of Early Intervention, 2020; 27(2): 182-194.

23. VERONEZ M, et al. Vivência de mães de bebês prematuros do nascimento a alta: notas de diários de campo. Rev. Gaúcha Enferm., 2017; 38(2): e60911. 